\title{
Interactions of Cytoplasmic Dynein Light Chains Tctex-1 and LC8 with the Intermediate Chain $\mathrm{IC}^{\dagger} 4^{\dagger}$
}

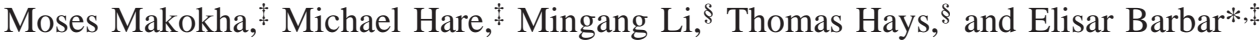 \\ Department of Chemistry and Biochemistry, Ohio University, Athens, Ohio 45701 and \\ Department of Genetics, Cell Biology and Development, University of Minnesota, \\ Minneapolis, Minnesota 55455
}

Received October 24, 2001; Revised Manuscript Received January 10, 2002

\begin{abstract}
The interactions of three subunits of cytoplasmic dynein from Drosophila melanogaster, LC8, Tctex-1, and the N-terminal domain of IC74 (N-IC74, residues 1-289), were characterized in vitro by affinity methods, limited proteolysis, and circular dichroism spectroscopy. These subunits were chosen for study because they are presumed to promote the assembly of the complex and to be engaged in the controlled binding and release of cargo. Limited proteolysis and mass spectrometry of N-IC74 in the presence of LC8 and Tctex-1 localized binding of Tctex-1 to the vicinity of K104 and K105, and localized binding of LC8 to the region downstream of K130. Circular dichroism, fluorescence, sedimentation velocity, and proteolysis studies indicate that N-IC74 has limited secondary and tertiary structure at near physiological solution conditions. Upon addition of LC8, N-IC74 undergoes a significant conformational change from largely unfolded to a more ordered structure. This conformational change is reflected in increased global protection of N-IC74 from proteolytic digestion following the interaction, and in a significant change in the CD signal. A smaller but reproducible change in the CD spectra was observed upon Tctex-1 binding as well. The increased structure introduced into N-IC74 upon light chain binding suggests a mechanism by which LC8 and Tctex-1 may regulate the assembly of the dynein complex.
\end{abstract}

Cytoplasmic dynein is a principal motor for minus enddirected intracellular transport along microtubules. While this protein complex participates in multiple cellular functions including mitosis and vesicular trafficking, its assembly and targeting to different cellular cargoes is not well understood. Cytoplasmic dynein is a multisubunit protein complex (1.2 $\mathrm{MDa}$ ) composed of two globular heads joined by flexible stalk domains to a common base (Figure 1). The head and stalk domains comprise the heavy chain motor subunits $(\sim 530 \mathrm{kDa})$ which contain the microtubule binding sites and the hydrolytic ATP-binding sites required for force production. The base of the motor complex contains two $74 \mathrm{kDa}$ intermediate chains (IC74), ${ }^{1}$ four light intermediate chains $(52-61 \mathrm{kDa})$, and several light chains $(10-25 \mathrm{kDa})(1-3)$. These accessory subunits are thought to mediate motor complex assembly and connection to the transported cellular cargo (4).

By comparison to the multiplicity of kinesin heavy chain genes, the diversity of cytoplasmic dynein heavy chains is

This work is supported by grants from Ohio Cancer Research Associates (E.B.), NIH GM60969 (E.B.), NIH GM44757 (T.S.H.), American Heart Established Investigator Award (T.S.H.) and American Cancer Society (M.H.).

* Corresponding author: Elisar Barbar, Tel: (740) 593-1751, Fax: (740) 593-0148, e-mail: barbar@ohio.edu.

$\stackrel{+}{*}$ Ohio University.

$\S$ University of Minnesota.

${ }^{1}$ Abbreviations: IC74, $74 \mathrm{kDa}$ dynein intermediate chain; N-IC74, C-IC74, N- and C-terminal domains of IC74; LC8, $10 \mathrm{kDa}$ dynein light chain (also referred to as PIN); Tctex-1, $12.5 \mathrm{kDa}$ dynein light chain (also referred to as LC14); ESI-MS, electrospray ionization mass spectrometry; CD, circular dichroism; DTT, dithiothreitol; IPTG, isopropyl- $\beta$-D-thiogalactoside.

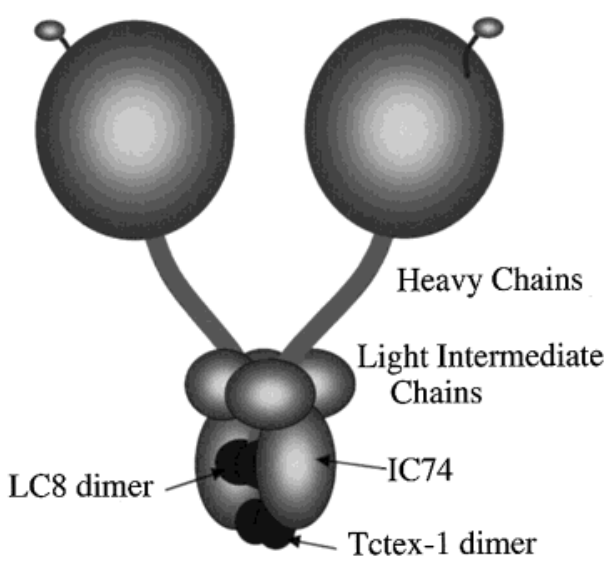

FIGURE 1: Schematic representation showing the relationship of the components of dynein to each other. This relation of the motor head and stalk domains to the light and intermediate subunits is based on images from electron microscopy (40). The base of the complex contains a number of light chains in addition to LC8 and Tctex-1. The association states of LC8 and Tctex-1 is determined from our work and others $(21,41-43)$.

quite limited. Only two cytoplasmic heavy chain genes have been well characterized, the predominant and ubiquitously expressed Dhc 1a isoform and the minor Dhe 1b isoform that appears to be more functionally restricted $(5-8)$. The limited diversity of the cytoplasmic dynein heavy chain has fostered the hypothesis that the multiple functions of dynein are mediated through variations in the composition, modification, and interactions of the intermediate, light intermediate, and light chains. The heterogeneity of these accessory subunits within the base of the motor complex could enable 
coupling of the dynein motor to a wide variety of intracellular cargo (1). Moreover, the utilization of accessory subunits within both axonemal and cytoplasmic dynein suggests they may also function in motor complex assembly. Further structural studies of the accessory subunits are needed to help elucidate the relationship between dynein motor structure and function.

The light chains LC8 (10 kDa), Tctex-1 (12.5 kDa), and the intermediate chain IC74 $(74 \mathrm{kDa})$ interact tightly as a subcomplex within the base of the native dynein complex (9). LC8 and Tctex-1 bind to a number of unrelated cargo molecules and are highly conserved among species. Between human and Drosophila, the sequence identities of LC 8 and Tctex-1 are 94 and $70 \%$, respectively $(1,10,11)$. IC74 binds to dynein heavy chain, linking the light chains to the heavy chains, and it also binds to dynactin $(4,12)$. The nature and regulation of the interaction between the IC74 and dynactin is of considerable interest. Dynactin is proposed to mediate the association of numerous cellular cargoes with the dynein motor and to further facilitate the processive movement of cargo along the microtubule (13-15).

The intermediate chain contains two functionally and structurally independent domains, as shown by truncation mutants of IC74 from Dictyostelium (16) and sequence-based structure prediction. Sequence analysis predicts six WD repeats in the $\mathrm{C}$-terminal domain, a motif known to form a $\beta$-propeller structure and promote the assembly of heteromeric protein complexes (17). Deletion analysis shows that the $\mathrm{C}$-terminal domain mediates the interaction between the intermediate chain and the heavy chain subunits (16). The C-terminal domain is highly conserved, with $71 \%$ identity between Dictyostelium and rat, and has the conserved function of binding to dynein heavy chain. The $\mathrm{N}$-terminal domain of IC74 is far less conserved, with only $21 \%$ identical residues in the corresponding Dictyostelium and rat sequences. However, multiple sequence alignments reveal a well-conserved stretch of amino acids in the N-terminal region, referred to as the PPE/TQT box (18). Successive deletions of mouse IC74 indicate that binding of LC8 to IC74 requires a KETQT sequence on the $\mathrm{N}$-terminal region, while binding of Tctex-1 requires a RRLNK sequence $(19,20)$. The binding site for dynactin is also found at the N-terminus, since its truncation prevents binding to the dynactin complex, while heavy chain binding is preserved (16).

We have initiated studies to fully characterize the interactions of accessory subunits located within the base of the Drosophila cytoplasmic dynein complex. In the long term, the ability to combine in vitro and in vivo studies of mutant subunits will facilitate our understanding of their contribution to motor assembly and function. In the present work, we expressed and purified LC8, Tctex-1, and N- and C-terminal domains of IC74 from Drosophila. Using limited proteolysis and mass spectrometry, we mapped the sites on IC74 that interact with LC8 and Tctex-1 at near physiological conditions. Spectroscopic and hydrodynamic characterization indicate that the free N-terminal domain of IC74 has limited regular secondary structure but gains structure and stability on binding to LC8. This large conformational change upon binding to LC8 may be important in assembly and regulation of the dynein complex.

\section{EXPERIMENTAL PROCEDURES}

Cloning, Overexpression, and Purification of LC8, Tctex1, and IC74 Constructs. LC8 was prepared as described previously (21). Tctex-1 was prepared according to the following procedure. The gene encoding Drosophila Tctex-1 was subcloned into the bacterial expression vector pTrcHis with an $\mathrm{N}$-terminal polyhistidine tag (Invitrogen). A protease Factor Xa recognition sequence was engineered immediately $5^{\prime}$ to the start codon of the cDNA clone. The PCR fragment was excised as a KpnI-HindIII fragment and cloned into the corresponding site of $\mathrm{pTrcHis}$ bacterial expression vector in Escherichia coli Top10 cell line (Invitrogen). Induction of expression with $0.4 \mathrm{mM}$ IPTG was performed at $25^{\circ} \mathrm{C}$ for about $12 \mathrm{~h}$. Crude extract was purified by affinity chromatography on Ni-nitrilotriacetate (Ni-NTA) agarose column (Qiagen Inc) by elution with $350 \mathrm{mM}$ imidazole. The fractions were dialyzed against $20 \mathrm{mM}$ Tris buffer $\mathrm{pH}$ 8.0 , containing $0.2 \mathrm{M} \mathrm{NaCl}$ and $2 \mathrm{mM} \mathrm{CaCl}_{2}$ and incubated with $1 \%$ Factor $\mathrm{Xa}$ for $9 \mathrm{~h}$ at $37^{\circ} \mathrm{C}$. Completion of the reaction was monitored by SDS-PAGE. Cleavage of the His tag by Factor Xa releases the native sequence of Tctex1. The protein was further purified from the fusion peptide and the protease on a macroprep DEAE anion-exchange resin (Bio-Rad) and eluted with $0.2 \mathrm{M} \mathrm{NaCl}$. Purity of $>95 \%$ was verified by SDS-PAGE and analytical size exclusion chromatography. The mass determined by electrospray ionization mass spectrometry was $12479 \mathrm{Da}$, in agreement with the calculated mass of 12478.2 Da.

The DNA encoding domains of Drosophila dynein intermediate chain were subcloned into the bacterial expression vector $\mathrm{pET} 15 \mathrm{~d}$ with an $\mathrm{N}$-terminal polyhistidine tag (Novagen). For construction of IC74 domains, wild-type cDNA (accession \# AF26337) was used as a template for the N- and C-terminal PCR deletion constructs. Synthesis of the N-terminal sequence $(1-289)$ and the C-terminal sequence (290-624) was achieved by PCR amplification with BamH1 and HindIII restriction sites. The constructs were ligated into pET 15d plasmid, and transformed into BL21(DE3) (Invitrogen) bacterial expression cell lines.

The recombinant BL21(DE3) cells for both $\mathrm{N}$ - and Cdomains were inoculated in a $1-\mathrm{L}$ LB medium containing ampicillin $(60 \mu \mathrm{g} / \mathrm{mL})$ from an overnight culture and grown at $37{ }^{\circ} \mathrm{C}$ to an OD 600 of $0.6-0.8$. IPTG was added to a final concentration of $1 \mathrm{mM}$, and the culture was incubated at $37{ }^{\circ} \mathrm{C}$ for another $4 \mathrm{~h}$. The cells were harvested by centrifugation at $4000 \mathrm{~g}$ for $30 \mathrm{~min}$ and resuspended into 40 $\mathrm{mL}$ of $0.1 \mathrm{M}$ sodium phosphate buffer $\mathrm{pH} 8$ containing 5 $\mathrm{mM} \beta$-mercaptoethanol, and $1 \mathrm{mM}$ PMSF, then lysed by sonication. After centrifugation of the sample, the pellet was solubilized in $8 \mathrm{M}$ urea, $0.1 \mathrm{M}$ sodium phosphate, $\mathrm{pH} 8$, and $10 \mathrm{mM} \beta$-mercaptoethanol by shaking at room temperature for $1 \mathrm{~h}$. Crude extract after centrifugation was purified by affinity chromatography on Ni-NTA resin (Qiagen) by elution in $\mathrm{pH} 4$ denaturing buffer. The proteins were refolded by extensive stepwise dialysis in refolding buffer $(50 \mathrm{mM}$ sodium phosphate, $\mathrm{pH} 7.4,2 \mathrm{mM}$ reduced glutathione, 0.2 $\mathrm{mM}$ oxidized glutathione, $0.1 \% \mathrm{NaN}_{3}, 50 \mathrm{mM} \mathrm{KCl}$, and $5 \%$ glycerol) containing 4, 2, and $0 \mathrm{M}$ urea, respectively. Further purification of N-IC74 was achieved by elution on a hydroxyapatite column (Bio-Rad) using a linear gradient of phosphate buffer $(10-400 \mathrm{mM})$ and $1 \mathrm{mM}$ benzamidine, $\mathrm{pH}$ 
7.2; N-IC74 elutes around $200 \mathrm{mM}$. SDS-PAGE gel electrophoresis of the purified domains of IC74 shows a single band close to the expected molecular mass. The molecular mass of N-IC74 was determined by mass spectrometry to be $33880 \mathrm{amu}$, in accord with the calculated mass of 33 879.2.

Biochemical Interactions. Ni-NTA precipitation of either LC8 or Tctex-1 with His-tagged IC74 N- and C- terminal domains was used to screen for binding. Excess C- and $\mathrm{N}-\mathrm{IC} 74$ proteins with the His tag intact were incubated with $\mathrm{Ni}$-NTA resin overnight at $4{ }^{\circ} \mathrm{C}$ to saturate the resin and prevent nonspecific binding to LC8 and Tctex-1. Tctex-1 or LC8, dialyzed in the same buffer $(50 \mathrm{mM}$ sodium phosphate, $0.5 \mathrm{M} \mathrm{NaCl}, \mathrm{pH} 7.8$ ), were then added to the bound resin, and incubated at $4{ }^{\circ} \mathrm{C}$ for $8 \mathrm{~h}$. The resin was recovered by centrifugation, and after excessive washing, samples were resuspended in electrophoresis sample buffer and analyzed on a $15 \%$ SDS-PAGE gel, and visualized by Coomassie Brilliant Blue staining.

For coimmunoprecipitation, N-IC74 was incubated with equal amounts of purified LC8 or Tctex-1 or both in $0.1 \mathrm{M}$ sodium phosphate, $1 \%$ Triton- $100 \times, 0.3 \mathrm{M} \mathrm{NaCl}, \mathrm{pH} 7.8$ buffer. The mixtures were then treated with $5 \mu \mathrm{g}$ of mouse monoclonal antibody anti-IC74 (Chemicon International) and incubated overnight at $4{ }^{\circ} \mathrm{C}$. Protein $\mathrm{G}$ Agarose was incubated with the mixture for $3 \mathrm{~h}$. The immunoprecipitates were collected by centrifugation, washed extensively, then resuspended in electrophoresis sample buffer and analyzed by SDS-PAGE.

Limited Proteolysis. Limited proteolysis experiments were carried out using the enzymes trypsin (Promega), and proteinase $\mathrm{K}$ (Novagen). All reactions were performed in $50 \mathrm{mM}$ sodium phosphate buffer, $\mathrm{pH}$ 7.8. N-IC74 at a concentration of $13 \mu \mathrm{M}$ was incubated for $3 \mathrm{~h}$ at $4{ }^{\circ} \mathrm{C}$ in the presence and absence of equimolar concentration of LC8 or Tctex-1 or both in a final reaction volume of $100 \mu \mathrm{L}$. The samples were then treated with modified trypsin (enzyme substrate ratio 1:150) or proteinase $\mathrm{K}$ (enzyme substrate ratio 1:400), and incubated at $25^{\circ} \mathrm{C}$. The protease concentrations were chosen such that the small differences in the digestion of the free and bound N-IC74 were maximized. At 3-5 min intervals, $10 \mu \mathrm{L}$ aliquots were removed and treated with the protease inhibitor phenylmethanesulfonyl fluoride (Sigma) to a final concentration of $2 \mathrm{mM}$. Samples were run on a tricine SDS-PAGE gel (22), and visualized by silver staining. Protein substrates and proteases were prepared according to published methods (23).

For large-scale digestion, the reaction was scaled up 10fold to $1 \mathrm{~mL}$, and incubated for $30 \mathrm{~min}$ at $25^{\circ} \mathrm{C}$. The product was passed on a $\mathrm{C} 18$ Waters YMC-Pack reversed phase $250 * 6.0 \mathrm{~mm}$ column, and fractions corresponding to the protected fragments were identified by tricine SDS-PAGE and analyzed by mass spectrometry.

Mass Spectrometry and Identification of Fragments. Mass spectrometric analyses were performed using electrospray ionization mass spectrometry on a Bruker Esquire system, and the data were analyzed using Bruker DataAnalysis 2 software. To identify proteolytic fragments of N-IC74, the mass of the fragment was compared with all possible sequences within N-IC74 which are calculated to have the same mass ( $\pm 1 \mathrm{amu})$. When trypsin was used, the sequence search was limited to tryptic fragments. Since the possibilities are limited by the selectivity of the enzyme, in each case only one sequence was found to have a mass within experimental error of the observed mass. When the nonselective enzyme proteinase $\mathrm{K}$ was used, the observed mass of the protected fragment was found to correspond to several possible sequences within N-IC74. To positively identify the protected fragment, the purified fraction was subjected to further digestion with trypsin. The tryptic fragments, identified as above by mass spectrometry, were found to correspond to only one of the possible sequences of the larger fragment.

In several cases, a considerable discrepancy was found between the mass of N-IC74 fragments as determined by SDS-PAGE and by mass spectrometry. For example, a protein with a mass of 13973 was found at slightly higher than $M_{\mathrm{r}} 20 \mathrm{kDa}$ on the gel. This discrepancy can be explained by the high proportion of negatively charged residues in this fragment (15\%), which can restrict the binding of SDS and result in overestimation of the mass (24).

Circular Dichroism and Fluorescence Spectroscopy. Circular dichroism (CD) spectra were obtained using a JASCO 715 spectropolarimeter equipped with a Peltier type cell holder (model PFD-335S) which permits accurate temperature control. A built-in magnetic stirrer allows for fast equilibration within the cell. For thermal denaturation experiments, protein samples at a concentration of $3 \mu \mathrm{M}$ in $10 \mathrm{mM}$ phosphate buffer at $\mathrm{pH} 7.8$, were recorded in the far UV CD using a $1-\mathrm{cm}$ cell. Low protein concentration is used to prevent aggregation at high temperature. For $\mathrm{GdnCl}$ unfolding, experiments were recorded in $1-\mathrm{mm}$ cell at a protein concentration of $8 \mu \mathrm{M}$ in the far UV CD. Data were acquired at $25{ }^{\circ} \mathrm{C}$ using a batch-type experiment to ensure that equilibrium is achieved. Samples were equilibrated for $4 \mathrm{~h}$ at room temperature prior to acquisition. Spectra were corrected by subtraction of the corresponding spectra of blank samples.

Fluorescence emission spectra of the intrinsic tryptophan were determined on a Jobin Yvon/Spex spectrofluorometer. The excitation wavelength was set to $280 \mathrm{~nm}$, and fluorescence emission spectra were scanned from 300 to $380 \mathrm{~nm}$.

To monitor interactions by $\mathrm{CD}$, the spectrum of a stoichiometric mixture of two proteins (experimental) was compared to the sum of the individual spectra of the proteins (model for no interaction). All experiments were conducted at $25{ }^{\circ} \mathrm{C}$ with protein concentrations of $7 \mu \mathrm{M}$ in a $1-\mathrm{mm}$ path length cell. For the mixing experiment, molar ellipticity was calculated using a protein length and concentration corresponding to the average of the two proteins (25).

Analytical Ultracentrifugation. Sedimentation velocity data was collected on a Beckman XL1 analytical ultracentrifuge at a speed of $50000 \mathrm{rpm}$ at $4{ }^{\circ} \mathrm{C}$ for $8 \mathrm{~h}$. Samples were placed into double sector interference cells with dialysis buffer as reference. Data were analyzed by the time derivative method as discussed in ref 26. For a single homogeneous species, the sedimentation and translational diffusion coefficient are obtained from the peak position, and the width in a plot of $g\left(s^{*}\right)$ versus $s^{*}$. The molar mass and the frictional coefficient are calculated from the Svedberg equation (27).

Structure Prediction. Secondary structure prediction was determined using the PSIPRED method (28). The probability that a sequence will adopt a coiled-coil conformation was determined using the Coil program (29). The MultiCoil 


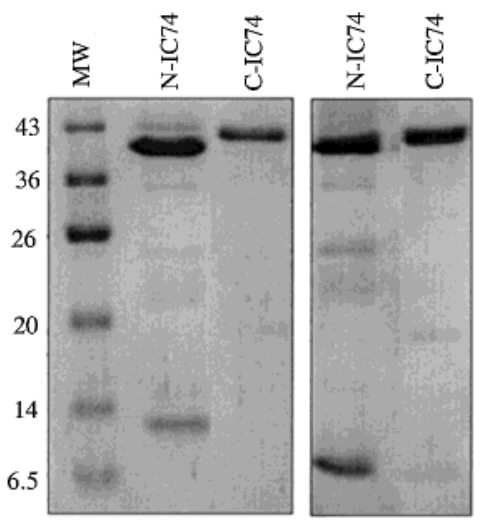

FIGURE 2: SDS-PAGE gels showing the interaction of LC8 (right) and Tctex-1 (left) with N-IC74 by Ni-NTA affinity precipitation. Binding was screened by mixing the light chains with Ni-NTA resin saturated with the His-tagged IC74 domain. After successive washing, the resin was mixed with SDS-PAGE buffer and run on a gel. LC8 and Tctex-1 were retained on the resin containing the $\mathrm{N}-\mathrm{IC} 74$, but were not retained by C-IC74. The gel was stained with Coomassie Brilliant Blue. Positions of molecular mass markers in $\mathrm{kDa}$ are shown on the left.

program was used to classify the coiled-coil predictions as dimeric or trimeric (30).

\section{RESULTS}

Interactions of Tctex-1 and LC8 with the N-Terminal Domain of IC74. Sequence analysis of IC74 predicts two independently folding domains: N-IC74, the N-terminal domain (residues 1-289, $33.9 \mathrm{kDa}$ ) and C-IC74, the Cterminal domain (residues 290-643, $41.2 \mathrm{kDa}$ ). The numbering is based on the full sequence from Genbank and excludes the 17 amino acids at the N-terminal that are part of the expression vector. The cDNA of the full IC74 Drosophila gene was used as a template for cloning these two domains into a pET expression vector with an $\mathrm{N}$-terminal 6-His tag to facilitate purification on a Ni-NTA agarose affinity column. Upon purification and refolding of both domains, we screened for interactions with LC8 and Tctex-1 using Ni-NTA precipitation and immunoaffinity methods. Excess 6-His tagged $\mathrm{N}$ - and C-terminal domains of IC74 were immobilized to a Ni-NTA affinity resin, and allowed to interact with either LC8 or Tctex-1. Since Tctex-1 and to a smaller extent LC8 bind slightly to the resin even in the presence of mild detergent and high salt, they were added after the resin was saturated with $\mathrm{N}-\mathrm{IC} 74$ to prevent nonspecific binding. The resin was then precipitated and analyzed by SDS-PAGE. Figure 2 shows SDS-PAGE gels of IC74 domains with LC8 (right) and Tctex-1 (left). A band for LC8 at $M_{\mathrm{r}} 8 \mathrm{kDa}$ and a band for Tctex-1 at $M_{\mathrm{r}} 12 \mathrm{kDa}$ are observed with $\mathrm{N}-\mathrm{IC} 74$. These bands represent a tight interaction of LC8 and Tctex-1 with N-IC74 that prevents their complete elution from the affinity resin after successive washing in high salt buffer. No bands corresponding to LC8 or Tctex-1 are observed with C-IC74 for experiments done at the same conditions. Interactions between N-IC74, LC8 and Tctex-1 were confirmed by coimmunoprecipitation experiments using a commercial anti-IC74 antibody. N-IC74 precipitates LC8 and Tctex-1 simultaneously (data not shown), indicating that both proteins bind to N-IC74, and that the binding of one does not inhibit the binding of the other.
Interactions Monitored by Limited Proteolysis and Mass Spectrometry. Regions of a protein that are in contact with another molecule can be identified by comparison of limited proteolytic digests of the free and interacting proteins. A site that is relatively seldom cleaved in the interacting protein is presumably close in the sequence to the binding site.

Figure 3 shows the sequence of N-IC74, with the location of the 37 potential trypsin cleavage sites indicated in bold. ESI-MS was used to determine the subset of these sites which are cleaved by trypsin under the conditions of these experiments. These are underlined in Figure 3. Conditions for limited proteolysis of N-IC74 in the presence of Tctex-1 or Tctex-1 and LC8 were determined by monitoring the progress of digestion with time on a tricine SDS-PAGE gel. Figure 4 shows a gel of N-IC74 after digestion with trypsin in the presence and absence of Tctex-1. Aliquots of both digests were taken and quenched at 5-min intervals. Comparison of the free and bound $\mathrm{N}-\mathrm{IC} 74$ shows that digestion is slower in the bound relative to the free protein. After 2030 min, virtually no large fragments of free N-IC74 are seen on the gel, while several bands indicative of protected cleavage sites are visible in lanes corresponding to bound $\mathrm{N}-\mathrm{IC} 74$. Uncleaved trypsin sites in these fragments are assumed to be protected from proteolysis by ligand binding. A single band at $M_{\mathrm{r}} 8 \mathrm{kDa}$ was chosen for analysis because of the clear difference in its appearance in the presence and absence of Tctex-1. This band (indicated by arrow in Figure 4) persists for over $30 \mathrm{~min}$ in the presence of Tctex-1, while in the digest of free IC74 the band has disappeared after 15 min. The mass of this fragment was determined to be 8169.9 Da by electrospray mass spectrometry after purification by HPLC. This mass corresponds to a tryptic fragment incorporating residues $53-130$ of N-IC74. There are three possible trypsin cleavage sites within this fragment: K104, K105, and K123. Since K123 is not cleaved by trypsin (Figure 3), most likely because of the neighboring proline residues P121 and $\mathrm{P} 122$, the protected residues within this sequence are K104 and K105.

When both LC8 and Tctex-1 are present, an abundant new fragment is observed with $M_{\mathrm{r}}$ slightly higher than $20 \mathrm{kDa}$ (indicated by an arrow in Figure 5). After purification by HPLC (Figure 6A, peaks 1 and 2) and identification by SDS-PAGE (Figure 6B), the mass of the larger fragment was determined by ESI-MS to be 13973 Da. This mass corresponds to a tryptic fragment incorporating residues 53183 (Figure 7A). This fragment contains K130 in addition to the missed cleavages present in the 8169.9 Da fragment. The 53-183 fragment is clearly present even after all other fragments are completely digested and only present when both LC8 and Tctex-1 are added. The 53-130 fragment with mass 8169.9 that is most protected with Tctex-1 is also observed in the digest of the LC8/Tctex-1/N-IC74 mixture (Figure 6B, lane 2). Digestion of N-IC74 in the presence of LC8 alone does not produce fragments that clearly result from direct protection. However, the protection of K130 in the presence of Tctex-1 and LC8 (but not with Tctex-1 alone) indicates that the binding site of LC8 is in this vicinity. In both cases, $\mathrm{N}-\mathrm{IC} 74$ is far more susceptible to rapid cleavage as compared to LC8 and Tctex-1. The bands that appear during this time period are all fragments of $\mathrm{N}-\mathrm{IC74}$, since neither LC8 nor Tctex-1 is perceptively cleaved under the conditions of this experiment (Figure 5, right). 


\begin{tabular}{|c|c|c|c|c|c|}
\hline RKR $\bar{D} L D E M L S S$ & $\overline{L G V A P V S E V L}$ & SSLSSVNSMT & SDNSNTQTPD & ASLQATVNGQ & $(100)$ \\
\hline SGG $\underline{\mathbf{K K}} \mathrm{PLNL}$ & SVYNVQATNI & PPKETLVYT్ㅡ & QTQTTSTGGG & NGDAHATDYY & $(150)$ \\
\hline DEYNLNPGLE & WEDEFTDDEE & SSLQNLGNGF & TSKূLPPGYLT & HGLPTV트VA & $(200)$ \\
\hline PAITPLEI $\underline{\underline{K K}}$ & 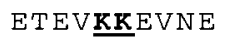 & 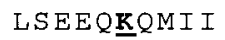 & LSENFQ $\underline{\boldsymbol{R} F V V}$ & RAGR $\underline{\mathbf{R}} \vee \mathrm{E} \underline{\mathbf{R}} \mathrm{A} L$ & $(250)$ \\
\hline YTDY & EAN & $D E \underline{R} S H A \underline{R} L S L$ & C DERW & & \\
\hline
\end{tabular}

FIGURE 3: The sequence of Drosophila N-IC74 with numbering based on the full sequence from Genbank (accession \# AF26337). The 17 amino acids at the N-terminal that are part of the expression vector. Potential trypsin cleavage sites are indicated in bold. Verified cleavage sites are underlined.

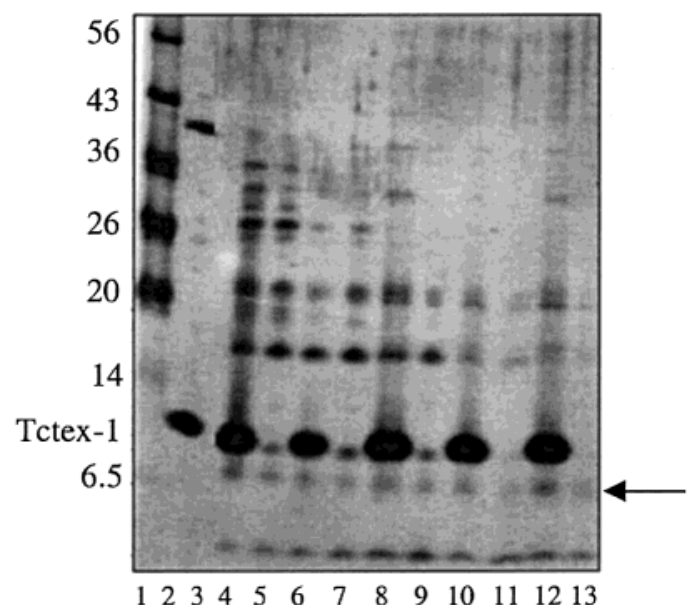

FIGURE 4: SDS-PAGE gel showing time dependent trypsin digestion of N-IC74 in the presence and absence of Tctex-1. Lane 1 contains molecular weight markers, lane 2 contains N-IC74 $\left(M_{\mathrm{r}}\right.$ $40 \mathrm{kDa})$ and Tctex-1 $\left(M_{\mathrm{r}} 12 \mathrm{kDa}\right)$, and lanes $4-13$ show tryptic digests of N-IC74 in the presence (even numbered lanes) and absence (odd lanes) of Tctex-1. Digestion time increases from left to right in 5-min intervals. The $M_{\mathrm{r}} 8 \mathrm{kDa}$ fragment indicated by an arrow is more protected in the presence of Tctex-1 than in free $\mathrm{N}-\mathrm{IC} 74$. The digestion was carried out at $25^{\circ} \mathrm{C}$, with enzymesubstrate ratio 1:150, and equimolar amounts of protein. The gel was visualized by silver staining

Similar results were obtained for limited digestion of $\mathrm{N}-\mathrm{IC} 74$ in the presence and absence of both LC8 and Tctex-1 using the nonspecific enzyme proteinase K. Comparison of proteinase $\mathrm{K}$ digests of free (Figure 8, lanes 2, 5, 8, and 11) and bound (lanes 4, 7, 10, and 13) N-IC74 shows that the band indicated by an arrow is more intense in the digest of $\mathrm{N}-\mathrm{IC} 74$ in the presence of Tctex- 1 and LC8, and after 15 min is observed only in the digest of the mixture. As with the tryptic digests, proteinase $\mathrm{K}$ digestion of Tctex- 1 and LC8 produced no observable fragments under these conditions (lanes 3, 6, 9, and 12). ESI-MS showed that the prevalent protected band was composed of three fragments, with masses 5060.8, 5274.2, and 5446.3. Since several sequences within N-IC74 can be found to match each of these masses, the isolated band was subjected to further digestion with trypsin. The major fragments in this digest were positively identified by mass as the tryptic fragments corresponding to residues $105-130$ and 106-130. Among the sequences which correspond to the masses 5060.8, 5274.2 , and 5446.3, only 99-147, 97-147, and 95-147, respectively, contain the sequence $105-130$. Therefore, we conclude that the presence of LC8 and Tctex-1 protects residues within the sequence $99-147$ of N-IC74 from cleavage by proteinase $\mathrm{K}$. This is in agreement with the finding that LC8 and Tctex-1 binding prevent trypsin cleavage at K104, K105, and K130.

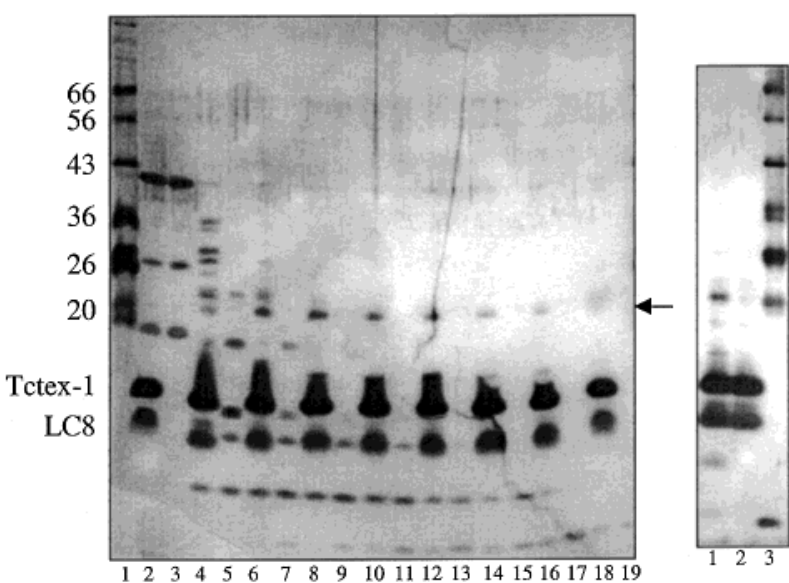

FIGURE 5: (Left) SDS-PAGE gel showing time dependent trypsin digestion of N-IC74 in the presence and absence of LC8 and Tctex1. Lanes $2-19$ show tryptic digests $\left(25^{\circ} \mathrm{C}\right.$, enzyme - substrate ratio $1: 150)$ of $\mathrm{N}-\mathrm{IC} 74$ in the presence (even numbered lanes) and absence (odd lanes) of equimolar amounts of both LC8 and Tctex1. Digestion time increases from left to right in 5-min intervals. The fragment appearing slightly higher than $M_{\mathrm{r}} 20 \mathrm{kDa}$ (indicated by an arrow) is protected in the presence of LC8 and Tctex-1. The 3 bands in lane 3 are due to nonspecific proteolysis of N-IC74 during the time it is removed from the inhibitor and before the digestion experiment starts. (Right) Lane 1 shows 30 min trypsin digestion of N-IC74 in the presence of LC8 and Tctex-1 (same as lane 15 on the left). Lane 2 shows 30 min trypsin digestion of LC8 and Tctex-1 with no N-IC74 present. Neither LC8 nor Tctex-1 produces any fragments under these conditions. Gels were visualized by silver staining.

In addition to longer lifetimes of fragments due to burial of cleavage sites at the interacting regions upon complex formation, global resistance to proteolysis is observed as well. Tryptic residues distant from the binding site may become inaccessible because of conformational changes that occur in the proteins following the interaction. Global protection from trypsin cleavage of N-IC74 is evident from the band for uncleaved N-IC74 (Figure 9, arrow) which persists longer in the presence of both LC8 and Tctex-1. Large fragments consistent with global protection are also observed with LC8 alone (data not shown), while no significant protection is observed with Tctex-1.

Physical Characterization of N-IC74. The strong signal at $203 \mathrm{~nm}$ and the weaker one at $222 \mathrm{~nm}$ in the far-UV CD spectra of N-IC74 indicate that the protein has some helical or coiled-coil structure, but also contains a large number of segments which lack regular secondary structure (Figure 10A). Similar spectra have been reported for disordered peptides with some helical content (31). Near-UV CD, which measures aromatic packing, shows no signal up to concentrations of $2 \mathrm{mg} / \mathrm{mL}$ in a $1-\mathrm{cm}$ cell (data not shown). A wavelength maximum of $\sim 350 \mathrm{~nm}$ in fluorescence emission 

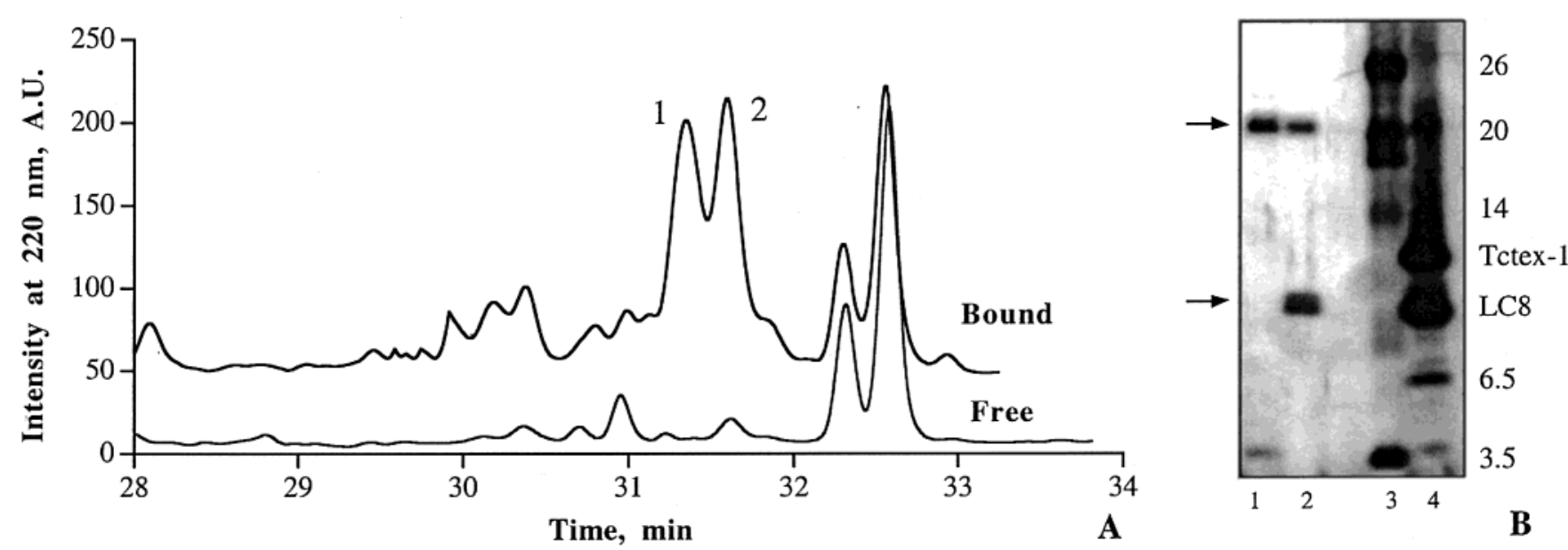

FIGURE 6: Purification of N-IC74 fragments by reversed-phase HPLC chromatography. (A) RP-HPLC analysis of the tryptic digest of N-IC74 after 30 min digestion at $25^{\circ} \mathrm{C}$ in the presence of both LC8 and Tctex-1 (top) and free (bottom). The peaks that appear in the presence of the ligands are labeled 1 and 2, and are eluted in $\sim 55 \%$ acetonitrile. (B) Lanes 1 and 2 are fractions corresponding to peaks 1 and 2. Lane 3 contains mass markers, with masses shown on the right. Lane 4 is the unpurified digest. The bands indicated by arrows are the $M_{\mathrm{r}} 20 \mathrm{kDa}$ fragment that shows most protection and intensity after 30 min of digestion and an $M_{\mathrm{r}} 8 \mathrm{kDa}$ fragment. The gel was visualized by silver staining.
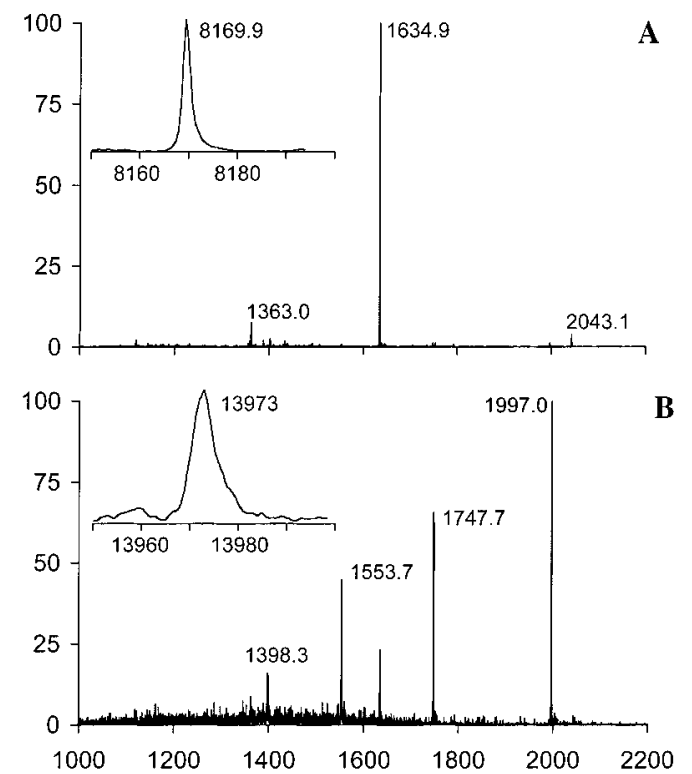

FIGURE 7: Identification of protected fragments of N-IC74 by ESIMS. Mass spectra of fragments 53-130 (A) and 53-183 (B). Fragments 53-130 and 53-183 migrate as $M_{\mathrm{r}} 8 \mathrm{kDa}$ and $20 \mathrm{kDa}$ on SDS-PAGE gel of Figures 5 and 6B. Insets show detail of spectrum after deconvolution of multiply charged peaks.

spectra indicates that Trp 161 and 289 are both solvent exposed (Figure 10B), and is consistent with minimal or absence of tertiary structure. Hydrodynamic analysis using sedimentation velocity is shown in Figure 10C. The time derivative plot $g\left(s^{*}\right)$ versus $s^{*}$ for $\mathrm{N}-\mathrm{IC} 74$ revealed a single peak, and when fit to a Gaussian function gave a sedimentation coefficient $s$ of $1.2 \mathrm{~s}$ and a translation diffusion coefficient $D$ of $4.07 \mathrm{~F}$. From these quantities, the molar mass was calculated to correspond to $25 \mathrm{kDa}$, somewhat smaller than a monomeric species. This, together with a frictional ratio of 2.6 indicates that N-IC74 is monomeric, and almost fully extended.

Far UV CD spectra were examined as a function of temperature and guanidinium chloride concentration to determine the degree to which these denaturants alter the limited secondary structure. Both temperature dependence

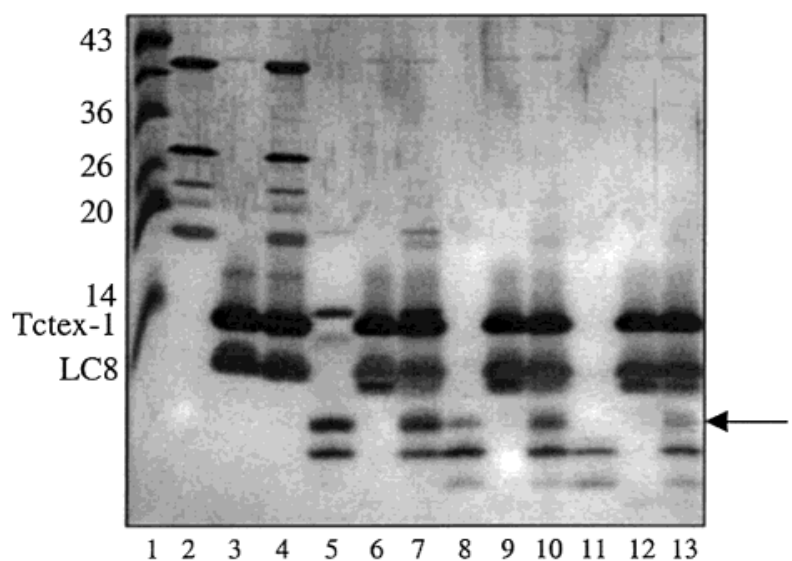

FIGURE 8: Proteinase K digests of N-IC74 in the presence of LC8 and Tctex-1 (lanes 4, 7, 10, and 13), with digestion time increasing from 0 to $15 \mathrm{~min}$ in 5-min intervals. Lanes $2,5,8$, and 11 are proteinase $\mathrm{K}$ digests of $\mathrm{N}-\mathrm{IC} 74$ alone, showing rapid degradation in the absence of the ligand. Lanes $3,6,9$, and 12 are proteinase $\mathrm{K}$ digests of LC8 and Tctex-1 (no N-IC74), showing that no observable digestion of these proteins occur in the time of the experiment. Digestion was carried out at $25^{\circ} \mathrm{C}$, with an enzymesubstrate ratio 1:400. The arrow points to an $M_{\mathrm{r}} 5 \mathrm{kDa}$ fragment which is protected by the presence of LC8 and Tctex-1.

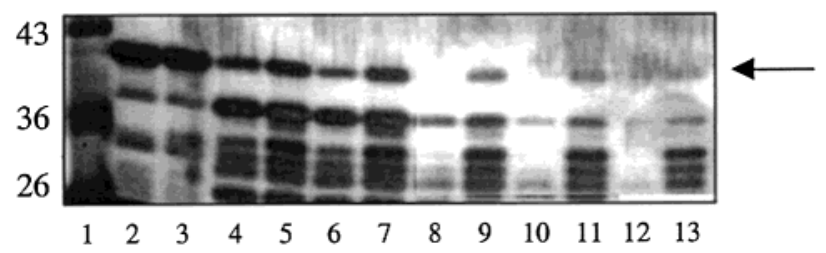

FIGURE 9: Trypsin digests of N-IC74 (lanes 2-13) in the presence (odd numbered lanes) and absence (even lanes) of LC 8 and Tctex1 , with digestion time increasing from left to right. This digest is similar to that shown in Figure 5, but was carried out with an enzyme-substrate ratio of 1:200 and shorter time intervals to emphasize early events. The intact N-IC74 band (arrow, $M_{\mathrm{r}} 40 \mathrm{kDa}$ ), and the high-mass fragments which persist only in the presence of LC8 and Tctex-1, indicate that the presence of the ligands affords protection to the entire protein by global stabilization.

over the range of 5 to $75^{\circ} \mathrm{C}$ (Figure 11A) and guanidinium chloride concentration range of $0-2 \mathrm{M}$ (Figure 11B) show that helicity is decreased in a noncooperative manner. 

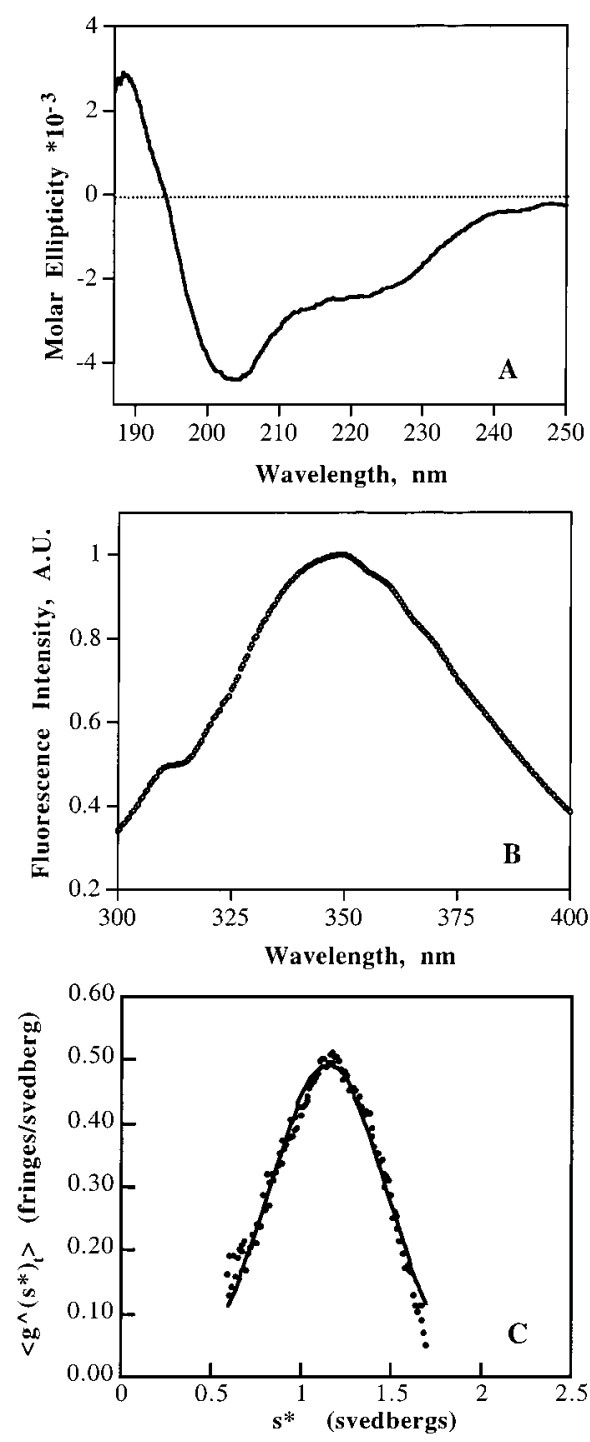

Figure 10: Physical characterization of N-IC74. (A) The CD spectrum obtained for $7 \mu \mathrm{M} \mathrm{N}$-IC74 in $10 \mathrm{mM}$ phosphate buffer, $\mathrm{pH} 7$, at $25^{\circ} \mathrm{C}$. The small signal at $222 \mathrm{~nm}$ and the stronger signal at $203 \mathrm{~nm}$ show that the protein has limited regular secondary structure. (B) Fluorescence spectrum obtained at similar conditions, showing an emission maximum at $\sim 350 \mathrm{~nm}$ indicating the absence of tertiary packing in the vicinity of the Trp residues. The excitation wavelength was set at $280 \mathrm{~nm}$, with excitation and emission slit widths of 3 and $6 \mathrm{~nm}$, respectively. The concentration of N-IC74 was determined using a molar absorbance coefficient at $280 \mathrm{~nm}$ of $22900 \mathrm{M}^{-1} \mathrm{~cm}^{-1}$. (C) Sedimentation velocity of N-IC74 domain at $4{ }^{\circ} \mathrm{C}$ shows a Gaussian function fit to the values for $s$ and $D$, and used to determine molecular mass.

Interactions Monitored by Circular Dichroism. CD spectra of N-IC74 were acquired in the presence and absence of LC8 and Tctex-1. The sum of the spectra of the free proteins (model for noninteracting proteins) was compared to the spectrum of a stoichiometric mixture. Figure 12A shows an overlay of spectra of LC8 (red), N-IC74 (blue), the computed sum of LC8 and N-IC74 (green), and the interacting mixture (black) in molar ellipticity vs wavelength. The major difference between the computed spectrum and the spectrum of the mixture is a strong band at $208 \mathrm{~nm}$ in the mixture spectrum indicating some increase in helical structure, presumably in N-IC74. This clear difference between the model for noninteracting proteins and the experimental mixture confirms that an increase or rearrangement in
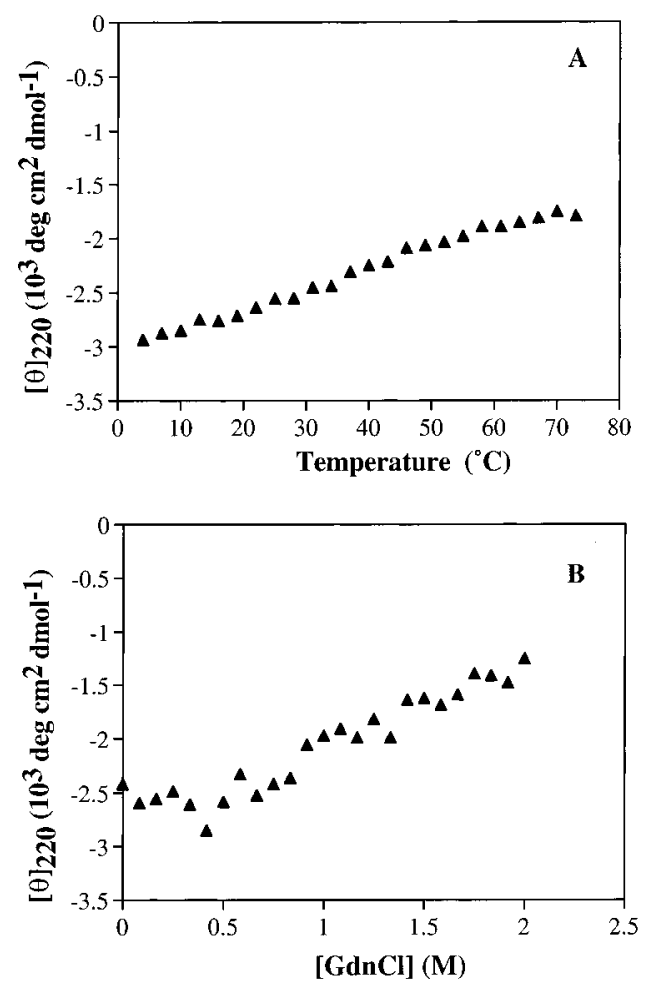

FIGURE 11: Unfolding profiles of N-IC74 as monitored by circular dichroism spectroscopy. Molar ellipticity of N-IC74 versus temperature (A), and guanidinium chloride concentration (B).

secondary structure accompanies the interaction of these two proteins. Similar experiments were performed in the presence of Tctex-1 instead of LC8, with Tctex-1 spectrum shown in red (Figure 12B). While there is some difference between the sum (green) and the mixture (black), the trend toward formation of a more helical structure is not as pronounced as in LC8. No difference was observed between the CD spectrum of a mixture of LC8 and Tctex-1 and the sum of their spectra taken separately (Figure 12C).

\section{DISCUSSION}

Both LC8 and Tctex-1 Bind to the N-terminal Domain of IC74. On the basis of secondary structure prediction, IC74 is comprised of two structurally independent folding domains. The N-terminal domain (which we refer to as N-IC74) is predicted to be disordered except for two short segments of coiled coil, and the C-terminal domain (C-IC74) is predominantly $\beta$-sheet with six WD repeats predicted to fold into a $\beta$-propeller. Truncation mutations of these domains show that they have independent functions: N-IC74 binds dynactin, while C-IC74 binds the heavy chain (16). In the present work, we have developed in vitro assays using purified recombinant polypeptides to investigate the interaction of the intermediate chain with the LC8 and Tctex-1 subunits. Using histidine fusion constructs of the IC polypeptide in affinity pull down experiments, we show that the $\mathrm{N}$-terminal domain (residues 1-289) of Drosophila IC74 interacts with both LC8 and Tctex-1. Neither light chain interacts with the C-terminal domain (290-643) of IC74. Independent coimmunoprecipitation assays further substantiate these interactions. An association between N-IC74 and both LC8 and Tctex-1 was predicted from experiments showing that these three subunits remain associated after salt-induced dissociation and sucrosegradient fractionation of dynein isolated from rat brain (9). 

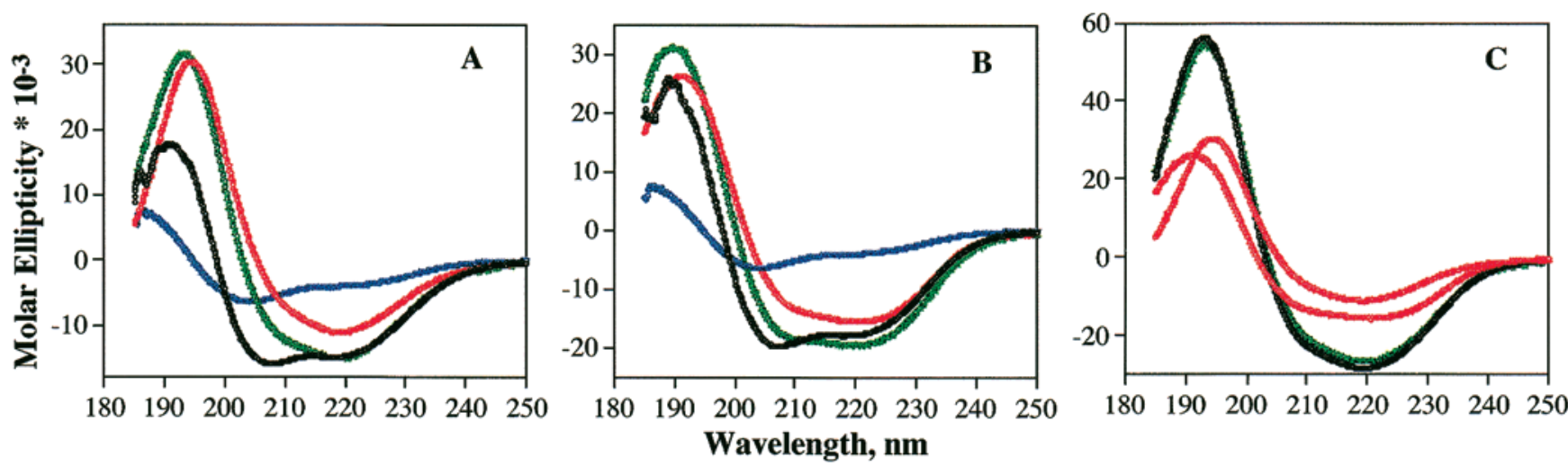

FIGURE 12: Changes in CD-detected structure upon interaction of N-IC74 with LC8 and Tctex-1. CD spectra for a mixture of N-IC74 at $7 \mu \mathrm{M}$ with equimolar concentration of LC8 (A), and Tctex-1 (B). N-IC74 spectra are shown in blue, LC8 and Tctex-1 in red, the spectra of the mixture in black, and the sum of individual spectra of the two proteins (model for no interaction) in green. A difference between the mixture and the sum indicates a conformational change upon interaction. The conformational change is more pronounced in the CD spectra of interactions with LC8 (A, comparison of green with black). Data for a mixture of LC8 and Tctex-1 are shown for comparison (C). In these spectra, there is no change between experimental (black) and calculated (green) consistent with the absence of interaction.

Mapping the Binding Sequences on IC74. Limited proteolysis using trypsin and proteinase $\mathrm{K}$ followed by ESIMS was used to locate specific cleavage sites in the N-IC74 sequence that are exceptionally well protected by association with LC8 or Tctex-1. This technique was developed for protein conformation studies $(23,32)$, and has also been used to investigate protein - protein complexes (33-35). In these experiments, proteolysis of a large domain of IC74 is administered under near-physiological conditions in the presence of the associating light chains. N-IC74 is far more susceptible to proteolysis than the light chains, and under the conditions of these experiments all of the proteolytic fragments are from N-IC74. After digestion, the protected $\mathrm{N}-\mathrm{IC} 74$ fragments were first identified on silver stained SDS-PAGE tricine gels by comparison with the digest of free N-IC74. The fragments were separated by HPLC, rerun on a gel for identification, and fractions containing protected fragments were analyzed by ESI-MS. In each case, the sequence of the tryptic fragments could be unambiguously identified from its mass. With proteinase $\mathrm{K}$, the sequence of protected fragments was identified by further digestion with trypsin to produce fragments easily identifiable by mass spectrometry.

Tctex-1 specifically protects IC74 cleavage sites K104 and K105 (Figure 4) to produce a persistent fragment corresponding to residues 53-130. This fragment also contains a third potential cleavage site, K123, but extensive mass spectrometric analysis shows that trypsin does not cleave at K123. In the presence of both LC8 and Tctex-1, we detect a prominent protected fragment that spans residues 53 to 183 , indicating that $\mathrm{K} 130$ is protected in addition to K104 and K105. Trypsin readily cleaves at K130 in the absence of the ligand and interestingly, K130 is also cleaved in the presence of LC8 alone. Since LC8 does not protect K130 in the absence of Tctex-1, but this site is protected when both are present, the binding sites of LC8 and Tctex-1 presumably surround K130, but neither ligand alone has sufficient steric bulk to protect it from trypsin cleavage.

A smaller fragment in the same region of N-IC74 was found to be protected from digestion by LC 8 and Tctex-1 when proteinase $\mathrm{K}$, a nonspecific protease with cleavage preference for hydrophobic aliphatic and aromatic sites, was used as the proteolytic enzyme. As with trypsin, limited proteolysis with proteinase $\mathrm{K}$ requires identification of protected fragments with SDS-PAGE, purification of the fragments with HPLC, and determination of the fragment masses. However, since proteinase $\mathrm{K}$ is a nonspecific protease, further digestion of fragments by trypsin was performed to identify the sequence of the fragments. Following this procedure, the fragments protected from proteinase K digestion were identified as $95-147,97-147$, and 99-147. The protected region from residues 99 to 147 contains K104, K105, and K130, consistent with the results of limited proteolysis with trypsin. These results also indicate that the segments protected by LC8 and Tctex- 1 coincide with the so-called PPE/TQT box (18), which refers to residues 121 to 123 and 132 to 134 in IC74. This region, unlike much of the $\mathrm{N}$-terminal domain, is highly conserved among species.

Our analysis of the protection of N-IC74 by LC8 and Tctex-1 using the Drosophila polypeptides implicates binding sites that are comparable to those recently suggested for the mouse IC74 polypeptide. M. Zhang and co-workers used successive deletions of IC74 to map the binding site of mouse LC8 and Tctex-1 on $\operatorname{IC74}(19,20)$. Although there is significant sequence identity between mouse and Drosophila LC8 (93\% identity), the similarity between Tctex-1 (70\%) and the N-terminal domain of IC74 (30\%) from the two species is considerably less. Given the difference in methodology used to characterize the interactions of these proteins from two different species, the results suggest interesting similarities. For LC8, they map the binding to the KETQT sequence, which in Drosophila corresponds to residues K130 to T134. This sequence is within the fragment of Drosophila $\mathrm{N}-\mathrm{IC} 74$ that we find is protected from cleavage when both LC8 and Tctex- 1 are present. For Tctex-1, they map the binding to the RRLNK sequence, which in Drosophila corresponds to residues K104 to N109, and is within the segment protected in the presence of Tctex-1.

The N-Terminal Domain Is Extended and Lacks Regular Secondary Structure. The N-terminal domain is a largely unfolded noncompact structure as shown by the absence of $\mathrm{CD}$ signal in the near $\mathrm{UV} \mathrm{CD}$, a fluorescence emission wavelength maximum of $\sim 350 \mathrm{~nm}$, and the relative ease of proteolysis as compared to other proteins. The noncooperativity observed in both temperature and denaturant profiles 
further confirms that N-IC74 does not behave as a folded globular protein. Far UV CD, however, indicates the presence of a small percentage of helical or coiled coil structure. MultiCoil, which specifically identifies coiled-coil forming sequences, predicts that residues $2-30$ have a propensity to form a dimeric coiled coil (30). Since hydrodynamic data show that N-IC74 is on average an elongated monomer, it is likely that N-IC74 forms a heterodimeric coiled-coil upon interaction with another protein such as dynactin which also has a coiled-coil domain.

LC8 Binding Increases Ordered Structure in N-IC74. On the basis of changes in circular dichroism spectra and a global increase in protection from proteolysis, it appears that a considerable increase in ordered structure in N-IC74 accompanies binding of LC8. Comparison of the far UV CD spectra of a mixture of N-IC74 and LC8 with the sum of LC8 and N-IC74 spectra before interaction (Figure 12A) shows a clear increase in signal at $208 \mathrm{~nm}$ indicating an increase in helical or coiled-coil structure in N-IC74 in the presence of LC8. Interaction with Tctex-1 produces similar effects, but to a much smaller extent (Figure 12B).

The persistence of intact N-IC74 and large fragments upon interaction with LC8 and with both ligands together (Figure 9) also suggests that LC8 binding induces an increase in structure or stability of N-IC74. Resistance to proteolysis has recently been shown to correlate with the extent of folding of apocytochrome $c$ upon binding to heme (36). Interaction with other molecules often increases the stability of proteins, so that the unfolding-folding equilibrium is shifted toward the folded conformation. This tends to decrease the effective exposure of the protein to the protease, without necessarily indicating a significant change in the secondary or tertiary structure of the protein. However, the changes we observe in the CD spectra of N-IC74 suggest that LC8 binding produces some increase in ordered structure in addition to increased stability. Tctex-1 does not significantly increase the amount of CD-detectable structure in $\mathrm{N}-\mathrm{IC} 74$, and does not apparently increase global protection from trypsin cleavage.

There are several examples of unstructured proteins that gain structure upon binding to their targets, most notably in proteins involved in cell cycle regulation and proteins which regulate transcription and translation as reviewed in ref 37. The plasticity afforded by the presence of a flexible structure with multiple conformations can allow a single protein to recognize a number of biological targets (38), and mediate different binding events (37). Lack of structure in the free state may also result in an increase in binding specificity, since the energetic cost of structural rearrangement must be compensated by a higher binding energy (39). A relatively disordered free state may be advantageous for regulatory proteins, as it would facilitate their removal by proteolysis when no longer needed. It is interesting to note that while the sequence of N-IC74 is not highly conserved among species (Drosophila N-IC74 has 23\% homology with Dictyostelium, $30 \%$ with mouse), the disorder predicted from their sequences is conserved. We speculate that the flexibility or disorder of this domain is a determinant of its specific interactions, and that a key event in formation of an active dynein complex is the simultaneous ordering of the IC74 structure and its binding with LC8.

\section{ACKNOWLEDGMENT}

The authors thank Dr. Knut Langsetmo for performing the sedimentation velocity experiment.

\section{REFERENCES}

1. King, S. M. (2000) Biochim. Biophys. Acta-Mol. Cell Res. $1496,60-75$

2. Susalka, S. J., Hancock, W. O., and Pfister, K. K. (2000) Biochim. Biophys. Acta-Mol. Cell Res. 1496, 76-88.

3. Gill, S., Cleveland, D., and Schroer, T. (1994) Mol. Biol. Cell 5,645 .

4. Steffen, W., Karki, S., Vaughan, K. T., Vallee, R. B., Holzbaur, E. L. F., Weiss, D. G., and Kuznetsov, S. A. (1997) Mol. Biol. Cell 8, 2077.

5. Criswell, P. S., Ostrowski, L. E., and Asai, D. J. (1996) J. Cell Sci. 109, 1891-1898.

6. Vaisberg, E. A., Grissom, P. M., and McIntosh, J. R. (1996) J. Cell Biol. 133, 831-842.

7. Pazour, G. J., Dickert, B. L., and Witman, G. B. (1999) J. Cell Biol. 144, 473-481.

8. Porter, M. E., Bower, R., Knott, J. A., Byrd, P., and Dentler, W. (1999) Mol. Biol. Cell 10, 693-712.

9. King, S. M., Barbarese, E., Dillman, J. F., Benashski, S. E., Do, K. T., Patel-King, R. S., and Pfister, K. K. (1998) Biochemistry 37, 15033-15041.

10. Tai, A. W., Chuang, J. Z., and Sung, C. H. (1998) J. Biol. Chem. 273, 19639.

11. Jaffrey, S. R., and Snyder, S. H. (1996) Science 274, 774.

12. Karki, S., and Holzbaur, E. L. F. (1999) Curr. Opin. Cell Biol. $11,45-53$.

13. Karki, S., and Holzbaur, E. L. F. (1995) J. Biol. Chem. 270, 28806-28811.

14. Vaughan, K. T., and Vallee, R. B. (1995) J. Cell Biol. 131, $1507-1516$.

15. King, S. J., and Schroer, T. A. (2000) Nat. Cell Biol. 2, $20-$ 24.

16. Ma, S., Trivinos-Lagos, L., Graf, R., and Chisholm, R. L. (1999) J. Cell Biol. 147, 1261-1273.

17. Garcia-Higuera, I., Gaitatzes, C., Smith, T. F., and Neer, E. J. (1998) J. Biol. Chem. 273, 9041-9049.

18. Nurminsky, D. I., Nurminskaya, M. V., Benevolenskaya, E. V., Shevelyov, Y. Y., Hartl, D. L., and Gvozdev, V. A. (1998) Mol. Cell. Biol. 18, 6816-6825.

19. Lo, K. W. H., Naisbitt, S., Fan, J. S., Sheng, M., and Zhang, M. J. (2001) J. Biol. Chem. 276, 14059-14066.

20. Mok, Y. K., Lo, K. W. H., and Zhang, M. J. (2001) J. Biol. Chem. 276, 14067-14074.

21. Barbar, E., Kleinman, B., Imhoff, D., Li, M., Hays, T., and Hare, M. (2001) Biochemistry 40, 1596-1605.

22. Schagger, H., and Vonjagow, G. (1987) Anal. Biochem. 166, $368-379$.

23. Carey, J. (2000) in Applications of Chimeric Genes and Hybrid Proteins, Pt C (Thorner, J., Emr, S. D., Abelson, S. D., Eds.) Academic Press, San Diego.

24. Takano, E., Maki, M., Mori, H., Hatanaka, M., Marti, T., Titani, K., Kannagi, R., Ooi, T., and Murachi, T. (1988) Biochemistry 27, 1964-1972.

25. Lawless-Delmedico, M. K., Sista, P., Sen, R., Moore, N. C., Antczak, J. B., White, J. M., Greene, R. J., Leanza, K. C., Matthews, T. J., and Lambert, D. M. (2000) Biochemistry 39, 11684-11695.

26. Stafford, W. F. (1992) Anal. Biochem. 203, 295-301.

27. Tanford, C. (1961) Physical Chemistry of Macromolecules, John Wiley \& Sons, Inc., New York.

28. Jones, D. T. (1999) J. Mol. Biol. 292, 195-202.

29. Lupas, A., Vandyke, M., and Stock, J. (1991) Science 252, $1162-1164$.

30. Wolf, E., Kim, P. S., and Berger, B. (1997) Protein Sci. 6, $1179-1189$.

31. Fezoui, Y., Hartley, D. M., Walsh, D. M., Selkoe, D. J., Osterhout, J. J., and Teplow, D. B. (2000) Nat. Struct. Biol. 7, 1095-1099. 
32. Brockerhoff, S. E., Edmonds, C. G., and Davis, T. N. (1992) Protein Sci. 1, 504-516.

33. Kriwacki, R. W., Wu, J., Siuzdak, G., and Wright, P. E. (1996) J. Am. Chem. Soc. 118, 5320-5321.

34. Fasshauer, D., Eliason, W. K., Brunger, A. T., and Jahn, R. (1998) Biochemistry 37, 10354-10362.

35. Scaloni, A., Monti, M., Acquaviva, R., Tell, G., Damante, G., Formisano, S., and Pucci, P. (1999) Biochemistry 38, 64-72.

36. Spolaore, B., Bermejo, R., Zambonin, M., and Fontana, A. (2001) Biochemistry 40, 9460-9468.

37. Wright, P. E., and Dyson, H. J. (1999) J. Mol. Biol. 293, 321331.

38. Kim, A. S., Kakalis, L. T., Abdul-Manan, M., Liu, G. A., and
Rosen, M. K. (2000) Nature 404, 151-158.

39. Spolar, R. S., and Record, M. T. (1994) Science 263, 777784.

40. Vallee, R. B., Wall, J. S., Paschal, B. M., and Shpetner, H. S. (1988) Nature 332, 561-563.

41. DiBella, L. M., Benashski, S. E., Tedford, H. W., Karrison, A., Patel-King, R. S., and King, S. M. (2001) J. Biol. Chem. $276,14366-14373$.

42. Liang, J., Jaffrey, S. R., Guo, W., Snyder, S. H., and Clardy, J. (1999) Nat. Struct. Biol. 6, 735-740.

43. Makokha, M., and Barbar, E. (2001) Biophys. J. 80, 2530.

BI011970H 